\title{
Reaction Rates in Chemically Heterogeneous Rock: Coupled Impact of Structure and Flow Properties Studied by X-ray Microtomography
}

\author{
Yousef Al-Khulaifi, ${ }^{\dagger, \neq}$ Qingyang Lin, ${ }^{*}{ }^{\dagger} \odot$ Martin J. Blunt, ${ }^{\dagger,+}$ and Branko Bijeljic ${ }^{\dagger,+}$
}

${ }^{\dagger}$ Department of Earth Science and Engineering and ${ }^{\ddagger}$ Qatar Carbonates and Carbon Storage Research Centre, Department of Earth Science and Engineering, Imperial College London, London SW7 2AZ, United Kingdom

\section{Supporting Information}

\begin{abstract}
We study dissolution in a chemically heterogeneous medium consisting of two minerals with contrasting initial structure and transport properties. We perform a reactive transport experiment using $\mathrm{CO}_{2}$-saturated brine at reservoir conditions in a millimeter-scale composite core composed of Silurian dolomite and Ketton limestone (calcite) arranged in series. We repeatedly image the composite core using X-ray microtomography (XMT) and collect effluent to assess the individual mineral dissolution. The mineral dissolution from image analysis was comparable to that measured from effluent analysis using inductively coupled plasma mass spectrometry (ICP-MS). We find that the ratio of the effective reaction rate of calcite to that of dolomite decreases with time, indicating the influence of dynamic transport effects originating from changes in pore structure coupled with differences in intrinsic reaction rates. Moreover, evolving flow and transport heterogeneity in the initially heterogeneous dolomite is a key determinant in producing a two-stage dissolution in the calcite. The first stage is characterized by a uniform dissolution of the pore space, while the second stage follows a single-channel growth regime. This implies that spatial memory effects in the medium with a heterogeneous flow characteristic (dolomite) can change the dissolution patterns in the medium with a homogeneous flow characteristic (calcite).
\end{abstract}

\section{INTRODUCTION}

Fluid-solid reactive transport in subsurface rock plays an important role in a number of applications such as carbon capture and storage (CCS), ${ }^{1}$ acidization in oil recovery, ${ }^{2}$ contaminant transport, ${ }^{3}$ and leaching. ${ }^{4}$ In CCS, long-term storage security remains a concern. ${ }^{1,5}$ The dissolution of $\mathrm{CO}_{2}$ into in situ brine forms carbonic acid, which in turn may cause a dissolution reaction with hosting carbonate formations. ${ }^{6,7}$ These reactive processes are dependent on rock mineralogy, which is a key control on the effective reaction rates from the pore to the field scales. 8 . There remains an uncertainty in the experimental estimation of reaction rates and kinetic input parameters for multimineral reactive transport models used for $\mathrm{CO}_{2}$ storage. ${ }^{10}$ Effective reaction rates have not only been shown to decrease with an increase in length scales ${ }^{11,12}$ but also are dependent on initial pore structure and flow heterogeneity. ${ }^{13,14}$ However, in multimineral systems, there is a complex coupling between transport and reaction that has not been experimentally investigated under reservoir conditions.

While continuum reactive transport models that can handle mixed mineralogy have been developed, ${ }^{15-20}$ most of these studies report a lack of validation in comparison to experimental measurements in realistic systems. Some also recognize the importance of observing pore-scale phenomena to better predict larger scale reaction rates and dynamic properties. ${ }^{19-21}$ Dissolution characteristics in consolidated rock have been well-characterized at the continuum scale, which is relevant for the fast reaction rates encountered in well stimulation, characterized by the short time scales of the acidizing process. ${ }^{22-24}$ Smaller scales are important in studying dissolution in lower reactivity media, as in $\mathrm{CO}_{2}$ storage processes, in which subtle changes in pore-scale connectivity control the evolution of reaction rate, porosity, and permeability. The impact of initial structure and transport conditions on dissolution has been studied for rocks of a single mineralogy using X-ray microtomography (XMT) by comparing scans prior to and after reaction. ${ }^{12,14,25-29}$ Menke et al. ${ }^{14,29}$ performed dynamic pore-scale imaging of calcite dissolution by $\mathrm{CO}_{2}$-saturated brine at reservoir conditions. They observed that an increase in initial pore structure heterogeneity favors dissolution in fast flow channels (channel growth), as opposed to uniform dissolution throughout the entire volume.

To understand the effect of mineralogy on effective reaction rates, a few studies have tackled dissolution in multimineral assemblages. $^{30,31}$ Smith et al. (2013) ${ }^{32}$ performed reaction experiments in which $\mathrm{CO}_{2}$-saturated brine was flowed through $15 \mathrm{~mm}$ diameter by $30 \mathrm{~mm}$ length reservoir core samples to explore their responses to variable partial pressures of $\mathrm{CO}_{2}$, initial pore structure, and calcite and dolomite content. They

Received: December 8, 2016

Revised: February 25, 2017

Accepted: March 13, 2017

Published: March 13, 2017 
used XMT to image the reactions and reported that greater pore space heterogeneity results in unstable dissolution fronts and, hence, much greater permeability increases.

In addition, several researchers have studied dissolution in rock cores with natural heterogeneous mineral patterns ${ }^{33-36}$ and constructed ones. ${ }^{37,38}$ These experiments provide evidence that the complexity of dissolution in natural systems stems from the fact that minerals with different intrinsic (batch) reaction rates are spatially distributed, resulting in different pore structures and flow/transport heterogeneities. This gives rise to the question of how to discriminate the pore structure, flow, and transport from reaction effects, which may profoundly change during dissolution. For this purpose, we design a composite rock system that consists of calcite and dolomite cores, for which we can initially control both chemical (from batch experiments, we know the minerals' intrinsic reaction rates) and physical (by characterizing the pore space and velocity field using X-ray microtomography and pore-scale modeling) heterogeneity. During dissolution in the two rock cores, both their effective reaction rates and their pore structures and velocity fields dynamically change, and we will be able to discriminate and quantify the coupling between pore structure, flow, and transport and the reactive properties. This will help us elucidate the nature of dissolution in complex mineral pore structures encountered in carbonate reservoirs, where, for example, there has been dedolomitization resulting in calcitized dolomite ${ }^{39}$ or other geological processes, which result in the layering and mixing of dolomite and calcite minerals at the millimeter to centimeter scales, ${ }^{40}$ and will allow us to quantify and explain the contributions and interplay between these reactive transport properties. Moreover, this can serve as a benchmark study for reactive transport models of chemically and physically heterogeneous media used in carbon storage, contaminant transport, and oil recovery.

To gain a deeper understanding of pore-scale phenomena in the presence of physical and mineral heterogeneity, we will: (a) measure effective reaction rates for a chemically heterogeneous composite system composed of two minerals having an order of magnitude difference in individual batch reaction rates and (b) study the impact of contrasting initial physical (flow and transport) heterogeneity in two minerals on the nature of the dissolution patterns. XMT has been widely used as a nondestructive imaging tool in geology-related studies. ${ }^{41-48}$ Reservoir-conditions imaging has been developed for studying supercritical $\mathrm{CO}_{2}$-brine systems in natural rock. ${ }^{49}$ The dynamic changes in porosity can be quantified for chemically homogeneous samples, either by means of in situ imaging with lab-based XMT or synchrotron sources. ${ }^{14,29}$ We use XMT to study reservoir-condition dissolution of a calcite and dolomite composite medium by $\mathrm{CO}_{2}$ dissolved in brine. We show how the coupling of different flow-transport characteristics and different intrinsic reaction rates of the two minerals impacts the effective reaction rates, permeability, and dissolution patterns. To achieve this, calcite (Ketton limestone) and dolomite (Silurian) were chosen for their contrasting reaction rates, pore structures, and flow heterogeneity characteristics. One important aspect of the work is to include effluent analysis. ${ }^{12,25-27,50}$ We also perform numerical analysis on the time-series of XMT images of the two-mineral composite medium by simulating flow through the pore space to quantify the changes in flow heterogeneity characteristics, such as velocity distributions. This enables us to examine how these characteristics evolve for both minerals, demonstrating the impact of dynamic coupling between transport and reaction on dissolution patterns.

\section{EXPERIMENTAL METHOD}

2.1. Rock Samples. We design the experiment by selecting two rock samples, Ketton limestone (calcite) and Silurian dolomite, which have highly contrasting flow, transport, and reaction characteristics. Calcite and dolomite are the most abundant carbonate reservoir minerals. ${ }^{51}$ Ketton limestone, which is $99 \%$ calcite, is from the Jurassic period and has a homogeneous pore structure made up of oolitic grains cemented together. ${ }^{52}$ In contrast, Silurian dolomite is $99 \%$ dolomite $^{53}$ and has a markedly more heterogeneous pore structure. The contrasting heterogeneity of the initial pore structures for the two rocks will be quantified in section 2.3 by their velocity distributions. We perform a dynamic reactive transport experiment with the two rock samples arranged in series. The batch reaction rate of the $\mathrm{CO}_{2}$-brine-calcite system under the same temperature and pressure conditions as in this work was measured to be $8.1 \times 10^{-4} \mathrm{~mol} /\left(\mathrm{m}^{2} \mathrm{~s}\right){ }^{6}$ Silurian dolomite batch reaction rates were measured to be 5.1 $\times 10^{-5} \mathrm{~mol} /\left(\mathrm{m}^{2} \mathrm{~s}\right)$, more than an order of magnitude lower than that of calcite. ${ }^{54}$ The Silurian sample is placed first in the sequence due to its significantly lower reaction rate compared to calcite; as a result, there should be a limited buffering of the acid when it reaches the Ketton sample.

We design our two-mineral layered porous medium (hereafter referred to as the composite core) by drilling $4.89 \mathrm{~mm}$ diameter cores of Silurian dolomite and a Ketton limestone and cutting them both to a length of $2.5 \mathrm{~mm}$. These cores are then stacked, giving a total length of $5 \mathrm{~mm}$; this length is required so that the complete core fits inside the field of view (FOV) for $\mathrm{XMT}$ when scanning at a $5 \mu \mathrm{m}$ voxel size. We apply a sufficiently high flow rate of $0.4 \mathrm{~mL} / \mathrm{min}$ that would render the effect of buffering of the acidic brine small due to the short relative length of the core and the continuous flow of fresh reactant. Figure S1 shows the full core composite assembly.

2.2. Experimental Methodology. The composite core is inserted into a Viton sleeve, which is placed into a carbon fiber Hassler type core holder (Figure S2B). A dry scan of the core is taken to image the initial conditions. The core is then flooded with 10 wt \% potassium iodide (KI) brine to purge the system of air. Then a $12 \mathrm{MPa}$ confining pressure is applied, and supercritical $\mathrm{CO}_{2}\left(\mathrm{scCO}_{2}\right)$ saturated brine $(5 \mathrm{wt} \% \mathrm{NaCl}$ and 1 wt $\% \mathrm{KCl})$ at $10 \mathrm{MPa}$ and $50{ }^{\circ} \mathrm{C}$ is injected into the core at a flow rate of $0.4 \mathrm{~mL} / \mathrm{min}$. The sequence of scans begins when the XMT projections show the highly attenuative KI brine leaving the pores of the core replaced by $\mathrm{scCO}_{2}$-saturated brine; we define this moment as $t=0$. A total of eight XMT scans were done, each having 901 projections, $100 \mathrm{keV}$ energy, a voxel size of $5 \mu \mathrm{m}$, and a duration of $45 \mathrm{~min}$; with this, changes in the pore structure can be captured without compromising image quality. We collected a $2 \mathrm{~mL}$ sample at the end of each scan which were analyzed in an ICP-MS (Agilent ICP-MS 7900) for $\mathrm{Ca}^{2+}$ and $\mathrm{Mg}^{2+}$ concentration for material balance calculations. Figure S2 shows the full experimental apparatus and a close-up of the core holder assembly along with a more detailed explanation on the experimental methodology.

2.3. Rock Heterogeneity Characteristics from Flow Simulation. We first quantify the difference in the two rock samples used by presenting intrinsic flow heterogeneity characteristics in the form of probability density functions (PDFs) of voxel velocities for Silurian and Ketton obtained 
from direct numerical simulation on the images of their pore space. We solve for volume conservation and the NavierStokes equations:

$$
\nabla \cdot u=0
$$

$$
\rho\left(\frac{\partial u}{\partial t}+u \cdot \nabla u\right)=-\nabla p+\mu \nabla^{2} u
$$

with a numerical finite volume solver implemented in OpenFOAM.$^{55,56}$ Pressure $(p)$ and velocity $(u)$ are obtained for each voxel between the inlet and outlet faces of the image; $\mu$ is the viscosity of water $(\mu=0.001 \mathrm{~Pa} \mathrm{~s})$, while $\rho$ is the density of water $\left(\rho=1,000 \mathrm{~kg} / \mathrm{m}^{3}\right)$. The Darcy velocity is calculated as $u_{\mathrm{D}}=\mathrm{Q} /\left(L_{y} L_{z}\right)$, where $Q\left(\mathrm{~m}^{3} / \mathrm{s}\right)$ is the total volumetric flux calculated as $Q=\int u_{x} \mathrm{~d} A_{x}, A_{x}\left(\mathrm{~m}^{2}\right)$ is the cross-sectional area perpendicular to the direction of flow $x$, and $\mathrm{u}_{x}$ is the face velocity normal to $A_{x} ; L_{x}, L_{y}$, and $L_{z}$ are the image lengths in each direction. The initial porosity of each mineral calculated from the XMT images $\left(\phi_{C T}\right)$ is 0.143 for Ketton and 0.099 for Silurian. Because in the flow simulation we only take into account the pore voxels that are connected along the axis parallel to flow $\left(\phi_{\text {connected }}\right)$, the resulting net porosities are 0.141 for the well-connected Ketton and 0.062 for Silurian. Permeabilities, $K$, were calculated at the core scale by dividing Darcy velocity by the average pressure gradient across the core and multiplying by viscosity, in accordance with Darcy's law: $K=-\mu \frac{u_{D}}{\nabla p}$.

The voxel velocities of the initial flow fields are presented by the red lines as part of the results in Figure 4 of section 3.3: panel A shows the normalized frequency of velocities of the composite medium, while panels B and C represent the Silurian and Ketton contribution, respectively, to the composite velocity frequency. Silurian and Ketton have highly contrasting flow heterogeneity characteristics. The Ketton velocity distribution shows a more narrow spread than that of Silurian: Ketton has a highly pronounced peak of velocities that are similar to the Darcy velocity, while the Silurian distribution is characterized by a large number of both stagnant and fast-flow voxels. This further confirms that Ketton is relatively homogeneous, ${ }^{57}$ whereas Silurian has a more heterogeneous flow characteristic. The connected porosity of the Silurian is less than half that of Ketton, and hence, it contributes a much smaller fraction to the overall velocity distribution in the composite.

2.4. Imaging and Effluent Analysis. All micro-CT images were reconstructed and an edge preserving nonlocal means filter is used to remove noise. ${ }^{58}$ Next, each image was segmented into two phases, rock and pore. Figure S3 shows the segmentation of the pores from rock samples with a seeded watershed algorithm using Avizo 9 software. ${ }^{59}$

Dissolution and transport are characterized by Damköhler (Da) and Péclet (Pe) numbers. The Damköhler number compares time scales for chemical reaction and mass transport $^{60}$ defined here as in Menke et al. (2015): ${ }^{29}$

$$
\mathrm{Da}=\frac{\pi r}{u_{\mathrm{av}} \times n}
$$

where $r$ is the reaction rate constant, $8.1 \times 10^{-4} \mathrm{~mol} /\left(\mathrm{m}^{2} \mathrm{~s}\right)$ in the case of calcite ${ }^{6}$ and $5.1 \times 10^{-5} \mathrm{~mol} /\left(\mathrm{m}^{2} \mathrm{~s}\right)$ for dolomite. ${ }^{54}$ The pore velocity $[\mathrm{m} / \mathrm{s}]$ is defined by $u_{\mathrm{av}}=u_{\mathrm{D}} / \phi_{\mathrm{CT}}$, where $\phi_{\mathrm{CT}}$ is the porosity measured from XMT images. $n$ represents the moles of mineral per unit volume of rock:

$$
n=\frac{\rho_{\text {mineral }}\left(1-\phi_{\text {total }}\right)}{M_{\text {mineral }}}
$$

where $\rho_{\text {mineral }}$ is the density $\left(2.82 \times 10^{3} \mathrm{~kg} / \mathrm{m}^{3}\right.$ measured on our dolomite sample and $2.71 \times 10^{3} \mathrm{~kg} / \mathrm{m}^{3}$ for calcite), and $M_{\text {mineral }}$ is the molecular mass $(0.1844$ and $0.1009 \mathrm{~kg} / \mathrm{mol}$, respectively). $\phi_{\text {total }}$ is the total porosity, including microporosity; how this is obtained is described later in the section. The Péclet number ${ }^{61}$ compares the effectiveness of mass transport of the solute by advection to that by diffusion and is expressed by

$$
\mathrm{Pe}=\frac{u_{\mathrm{av}} \times L_{\mathrm{c}}}{D_{\mathrm{m}}}
$$

where $D_{\mathrm{m}}$ is molecular diffusion coefficient $\left(7.5 \times 10^{-10} \mathrm{~m}^{2} / \mathrm{s}\right.$ for $\mathrm{Ca}^{2+}$ at $\left.25{ }^{\circ} \mathrm{C}\right) .{ }^{27}$ We calculate the characteristic length $L_{\mathrm{c}}$ (m) of consolidated rock samples from the XMT image specific surface area $S$ in $\left(\mathrm{m}^{-1}\right):{ }^{62}$

$$
L_{\mathrm{c}}=\frac{\pi}{S}
$$

The surface area was obtained by summing the number of voxel faces shared between grain and pore in the images. This area was then divided by the bulk volume to obtain the specific surface area, $S$. Calculated also is the diffusive Damköhler number $(\mathrm{PeDa})$ for each mineral, which relates reaction rate to diffusion rate obtained by multiplying $\mathrm{Pe}$ and $\mathrm{Da}$.

The effective reaction rate describes reaction rate in rock cores with irregular pore and velocity field structure, as opposed to the intrinsic or batch reaction rate under well-mixed conditions. Between consecutive XMT images, the effective reaction rate is calculated for each mineral individually using

$$
r_{\text {eff }}=\frac{\rho_{\text {mineral }}\left(1-\phi_{\text {micro }}\right)}{M_{\text {mineral }} S} \frac{\Delta \phi_{\mathrm{CT}}}{\Delta t}
$$

Here, $\rho_{\text {mineral }}$ is the density of pure mineral, and $\phi_{\text {micro }}$ is the subresolution porosity within the grains of the rock quantified through helium porosimetry discussed below, $\Delta \phi_{\mathrm{CT}}$ is the change in image calculated porosity between two scans a time $\Delta t$ apart, and $S$ is the specific surface area (calculated as described above) at the beginning of the considered time period. We calculate all these values for the calcite and dolomite portions of the core individually; these results are shown in Table S1.

We observe almost no change in gray scale values for solid voxels containing subresolution features for all scans indicating no measurable change in porosity in these regions. Because of the transport-dominant conditions caused by the relatively high flow rate of the experiment, surface reaction is favored over reaction in the intragranular porosity. In calculating the dissolved volume, $\Delta V_{\mathrm{d}}$, between scans for both minerals, the change in porosity was multiplied by the volume of the core, $V$, for each individual mineral: $\Delta V_{\mathrm{d}}=V \Delta \phi_{\text {total }}$. To account for the microporosity, nonresolvable by the micro-CT, we use

$$
\phi_{\text {total }}=\phi_{\text {micro }}\left(1-\phi_{\mathrm{CT}}\right)+\phi_{\mathrm{CT}}
$$

where $\phi_{\text {micro }}$ is calculated from helium porosimetry using

$$
\phi_{\text {micro }}=\frac{\phi_{\text {helium }}-\phi_{\mathrm{CT}}}{1-\phi_{\mathrm{CT}}}
$$



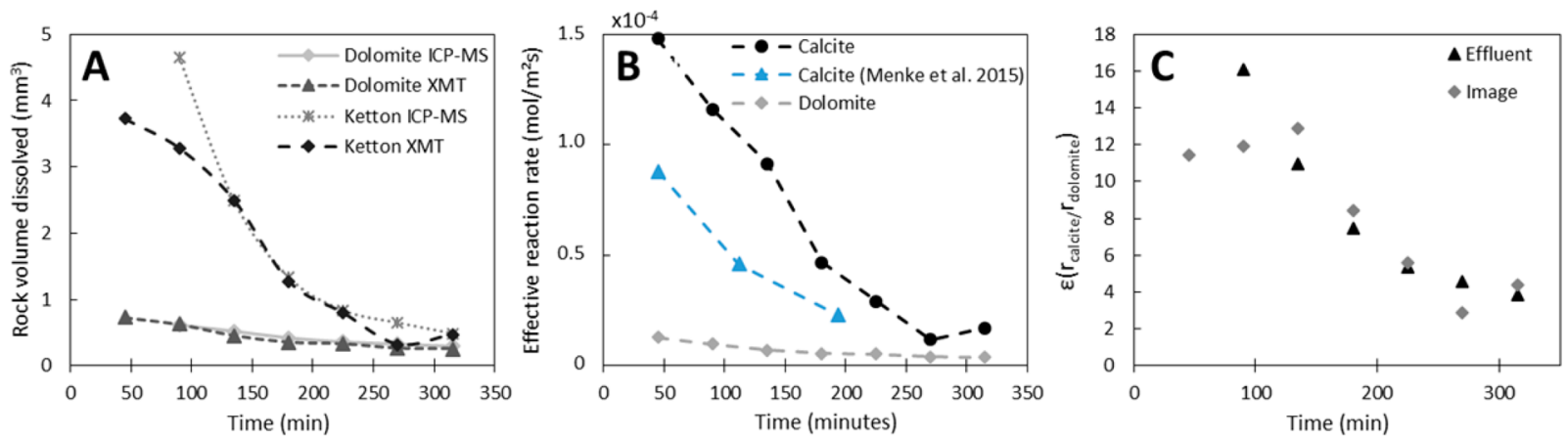

Figure 1. (A) Volume of calcite and dolomite dissolved in $45 \mathrm{~min}$ increments from Ketton and Silurian calculated from ICP-MS and from XMT images. A reasonable match is observed between the ICP-MS and XMT measurements. The first points from ICP-MS could not be quantified using this method because the initial effluent sample does not have representative concentrations due to the delay in the arrival of the reactive brine to the sampling point. (B) Effective reaction rates of Silurian and Ketton over the course of the dissolution experiment. Included on the plot is the effective reaction rate of Ketton from Menke et al. ${ }^{29}$ measured for a Pe ranging from 2100 to 1050 . (C) Plot of the ratio of reaction rates of dolomite and calcite calculated from ICP-MS effluent analysis $\left(\varepsilon_{\mathrm{e}}\right)$ and from micro-CT imaging $\left(\varepsilon_{\mathrm{i}}\right)$. The ratio steadily decreases with time because of muchgreater reduction in the calcite effective reaction rate.

where $\phi_{\text {helium }}$ is measured at the beginning of the experiment. The Ketton grain porosity was measured to be $\phi_{\text {micro }}=0.12,{ }^{63}$ and the Silurian grain porosity was measured to be $\phi_{\text {micro }}=$ 0.05 . Then, as dissolution proceeds, we assume that $\phi_{\text {micro }}$ remains the same and use eq 8 to estimate the total porosity as $\phi_{\mathrm{CT}}$ increases.

At the end of each scan, $2 \mathrm{~mL}$ of effluent is collected and the concentration of $\mathrm{Mg}^{2+}$ and $\mathrm{Ca}^{2+}$ in the sample is measured with ICP-MS. We assume that every dissolved mole of dolomite will give a mole of $\mathrm{Mg}^{2+}$ and $\mathrm{Ca}^{2+}$ each, based on the molecular formula of dolomite, $\mathrm{CaMg}\left(\mathrm{CO}_{3}\right)_{2}$. To find the moles of calcium from Ketton dissolution, we subtract the moles of $\mathrm{Ca}^{2+}$ coming from dolomite (equal to moles of $\mathrm{Mg}^{2+}$ ) from the total $\mathrm{Ca}^{2+}$ concentration of the sample. We find the volume dissolved of each mineral in the time between each two sampling points (45 min duration) by assuming a linear relationship between each measured concentration

$$
\Delta V_{n}=\frac{M_{\text {mineral }} \times Q\left(c_{n}+c_{n-1}\right)\left(t_{n}-t_{n-1}\right)}{2 \rho_{\text {mineral }}}
$$

where $n$ labels the sample number, $M_{\text {mineral }}$ is the molecular mass $(\mathrm{kg} / \mathrm{mol}), \Delta V$ is the volume dissolved $\left(\mathrm{m}^{3}\right), c$ is the mineral ion concentration of the sample in $\left(\mathrm{mol} / \mathrm{m}^{3}\right), t$ is the sampling time in $(\mathrm{s})$, and $Q$ is the flow rate in $\left(\mathrm{m}^{3} / \mathrm{s}\right)$. The volume of material dissolved at the end of the first $45 \mathrm{~min}$ period cannot be estimated accurately because $c_{0}=0$. Therefore, the point for calcite from ICP-MS at $45 \mathrm{~min}$ is omitted.

\section{RESULTS AND DISCUSSION}

We first provide XMT images and effluent analysis of dissolution in Silurian and Ketton to assess the dynamic changes in their individual effective reaction rates. Next we study the porosity-permeability relationship for Silurian, Ketton and the composite core. Furthermore, we examine the dynamics of flow and transport in each mineral core by visualizing flow fields and analyzing velocity distributions to observe and interpret the nature of time-dependent dissolution patterns in this composite system.

3.1. Effective Reaction Rates. Figure 1A shows the plots of rock volume dissolved between two successive scans for Silurian and Ketton calculated from XMT images and effluent analysis. There is a reasonable agreement in calculations of the rock volume dissolved from the measurements performed by ICP-MS and XMT for both minerals that constitute the composite core. These reaction rates are approximately 1 order of magnitude lower than that measured on initially flat surfaces without transport limitations, consistent with experiments on Ketton; $^{29}$ this indicates that even though we are in a high-Pe, low-Da regime (see Table S1), normally characterized as resulting in uniform dissolution, ${ }^{24}$ there are significant porescale transport limitations on the overall dissolution rate. Moreover, as we show later, the dissolution patterns are not all uniform, restricting reaction to only a subset of the pore space.

Figure $1 \mathrm{~B}$ shows the calculated effective reaction rates for both minerals in the time intervals between two consecutive scans. The effective reaction rates for the dolomite and calcite averaged over the course of our dynamic experiment are $6.92 \times$ $10^{-6}$ and $6.57 \times 10^{-5} \mathrm{~mol} /\left(\mathrm{m}^{2} \mathrm{~s}\right)$, respectively. As expected, the reaction rate for calcite is an order of magnitude greater than that for dolomite. While the reaction rate of the dolomite part of the composite core remained relatively steady during the experiment, the rate of calcite experienced a substantial drop, which then stabilized at late times. This suggests a large change in pore structure of Ketton during dissolution discussed later in section 3.2. In Figure 1B, we also compare our results with the effective reaction rates (the blue line) for a calcite-only dynamic experiment $^{29}$ in which the dissolution took place under similar transport conditions for a Ketton core of dimensions $4 \mathrm{~mm}$ in diameter by $120 \mathrm{~mm}$ in length. The $r_{\text {eff }}$ of Ketton in the composite core is higher than the $r_{\text {eff }}$ for the Ketton core in the calcite-only mineralogy experiment. The less reactive Silurian dolomite, which precedes Ketton in the composite core, shows a smaller buffering effect of the acidic brine, resulting in a higher reaction rate, compared to the Ketton only core, where images and calculations were performed on a $4 \mathrm{~mm}$ length section $2 \mathrm{~mm}$ away from the core inlet. Additionally, due to the lower reactivity in the preceding Silurian, less-acidic brine is consumed as compared to the calcite only core; this greater availability of unreacted acidic brine in Silurian increases the effective reaction rate in the Ketton part of the composite core, in comparison to the Ketton only case.

We define $\varepsilon$ as the ratio of the effective reaction rate of calcite to that of dolomite: 


$$
\varepsilon=\frac{r_{\text {calcite }}}{r_{\text {dolomite }}}
$$

The effective reaction rates for the dolomite and calcite averaged over the course of the experiment are different by an order of magnitude as mentioned above. We calculated $\varepsilon$ from image analysis, $\varepsilon_{\mathrm{i}}$, by taking the ratio of the effective reaction rates calculated from XMT scans and from the effluent, $\varepsilon_{\mathrm{e}}$. The ratio from effluent analysis was calculated as follows:

$$
\varepsilon_{\mathrm{e}}=\frac{\Delta V_{\text {calcite }}}{\Delta V_{\text {dolomite }}} \frac{\rho_{\text {calcite }}}{\rho_{\text {dolomite }}} \frac{M_{\text {dolomite }}}{M_{\text {calcite }}} \frac{S_{\text {dolomite }}}{S_{\text {calcite }}}
$$

$M$ is the molecular mass of the minerals, $S$ is the specific surface area calculated from the XMT image at the beginning of the time interval considered, and $\Delta V$ is the change in rock volume between scans, eq 10 . Figure $1 \mathrm{C}$ shows a plot of both $\varepsilon_{\mathrm{e}}$ and $\varepsilon_{\mathrm{i}}$ against time.

At early time ( $t=90 \mathrm{~min}), \varepsilon_{\mathrm{i}}$ and $\varepsilon_{e}$ are 12.0 and 16.1, respectively, and at intermediate time ( $t=180 \mathrm{~min}), \varepsilon_{\mathrm{i}}$ and $\varepsilon_{e}$ are 8.40 and 7.45 . Finally, at late time $(t=315 \mathrm{~min}), \varepsilon_{\mathrm{i}}$ and $\varepsilon_{e}$ become 4.40 and 3.87. Both $\varepsilon_{\mathrm{i}}$ and $\varepsilon_{e}$ show a decreasing trend. The decrease in reaction ratio is mainly due to the Ketton reaction rate becoming much lower, as the dissolution in Ketton changes from uniform to the dominant channel regime; this will be shown in the following section. The larger discrepancy between $\varepsilon_{\mathrm{i}}$ and $\varepsilon_{e}$ at early time could be explained by uncertainties in the segmentation of small differences in rock structure around grains that are dissolving.

3.2. Porosity-Permeability Relationship. From the segmented images, the porosity was calculated individually for the Silurian and Ketton part of the core at each scan time by counting pore and solid voxels. Permeability was obtained from flow simulations as described in section 2.3. The connected image porosity $\left(\phi_{\text {connected }}\right)$ versus permeability relationship are shown in Figure 2; the time evolution of the image porosity is displayed in Figure S4.

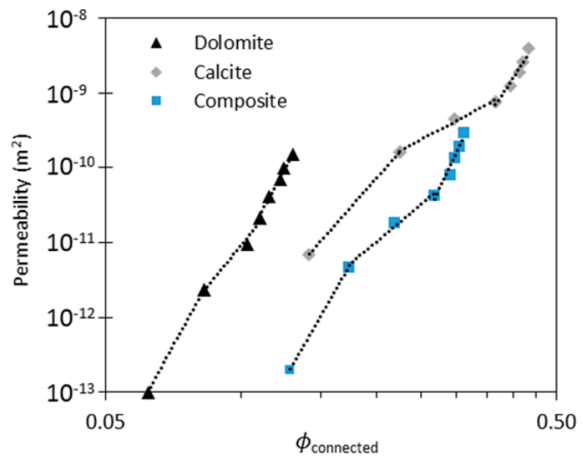

Figure 2. Calculated permeabilities for the entire core compared to those of the individual rock sections as a function of porosity plotted on a $\log -\log$ scale. Every data set is divided into three sections: $0-45$, $45-135$, and $135-315 \mathrm{~min}$, each of which is fitted with a power law trend, Table 1.

The trend in permeability and porosity for the composite medium is similar to that in Silurian dolomite, albeit with a shift to higher porosity, which is the contribution from the Ketton section to the composite core. This implies that resistance to flow of the composite system depends primarily on the resistance in the lower permeability Silurian. We observe different exponents $m$ within different regions of the porosity- permeability relationship when fitting the similarly sloped sections using a power law trend in line with $K=c \phi_{\text {connected, }}^{m}$ where $K$ is the permeability $\left(\mathrm{m}^{2}\right)$ and $c$ a constant $\left(\mathrm{m}^{2}\right)$. The variability in $m$ reflects transitions to different dissolution regimes, ${ }^{25}$ and higher $m$ values indicate a greater sensitivity of permeability to changes in porosity. Although in a previous study, the porosity-permeability trend of a homogeneous rock could be fitted with a single power law relationship; ${ }^{29}$ in our Silurian, Ketton, and composite data, we observe different scaling in the porosity-permeability relationship for different ranges of experimental times (see Figure 2 and Table 1): (i)

Table 1. Porosity-Permeability Power Law Fit Constants $c$

\begin{tabular}{|c|c|c|c|c|c|c|}
\hline \multirow{2}{*}{$\frac{\begin{array}{c}\text { time } \\
\text { period }\end{array}}{(\min )}$} & \multicolumn{2}{|c|}{ Dolomite } & \multicolumn{2}{|c|}{ Ketton } & \multicolumn{2}{|c|}{ composite } \\
\hline & $c\left(\mathrm{~m}^{2}\right)$ & $m$ & $c\left(\mathrm{~m}^{2}\right)$ & $m$ & $c\left(\mathrm{~m}^{2}\right)$ & $m$ \\
\hline $0-45$ & 2.3 & 11.1 & $4.0 \times 10^{-6}$ & 6.8 & $4.0 \times 10^{-4}$ & 10.4 \\
\hline $45-135$ & 0.0002 & 7.4 & $2.0 \times 10^{-8}$ & 3.2 & $3.0 \times 10^{-8}$ & 5.0 \\
\hline $135-315$ & 1.6 & 11.3 & $8.0 \times 10^{-6}$ & 9.3 & $8.0 \times 10^{-4}$ & 12.8 \\
\hline
\end{tabular}
and Exponents $m$

between $t=0$ and $t=45 \mathrm{~min}$, (ii) between $t=45 \mathrm{~min}$ and $t=$ $135 \mathrm{~min}$, and (iii) between $t=135 \mathrm{~min}$ and $t=315 \mathrm{~min}$. For the early-range (i) and late-range (iii) experimental times, the power-law exponent $m$ for the composite is close to that of Silurian. Another noteworthy observation is the changing of Ketton's $m$ exponent from 6.8 at early time to 9.3 at late time, signifying a shift to a more-sensitive permeability to porosity relationship due to the evolving heterogeneous flow field of the preceding Silurian dolomite. This observation implies different stages of dissolution, the nature of which will be studied in more detail in the following section.

3.3. Impact of Flow and Transport on Dissolution. To examine the impact of time-dependent changes of flow and transport characteristics on the nature of dissolution in the composite core, we visualize the normalized PDF of velocity for the composite images for scan times $t=0,90,180,270$, and $315 \mathrm{~min}$. Figure 3 presents the ratios of the magnitude of the velocity $(u)$ in each voxel to the Darcy velocity $\left(u_{\mathrm{D}}\right)$ on a linear color scale in which the lowest velocities are in blue and the highest velocities are in red. The two parts of the composite core have two different values for the average pore velocity due to the difference in porosities. Therefore, the velocities in Figure 3 were normalized by Darcy velocity, as this is fixed in the experiments. The isolated pores and stagnant regions are invisible as their voxel velocities are virtually zero.

It is seen in Figure 3 that a few preferential flow paths exist in the dolomite at early times, which is in accordance with its heterogeneous initial flow characteristic. These flow paths grow until $t=90 \mathrm{~min}$, when the establishment of a dominant flow channel occurs, which then continues to enlarge further throughout the experiment. However, dissolution in the Ketton calcite differs from that in the dolomite in two ways: first, at early times (until $t=90 \mathrm{~min}$ ), dissolution occurs moreuniformly in a large number of higher-velocity channels throughout the sample volume; this is expected considering different initial structures and the corresponding flow fields. However, at later times after $t=135 \mathrm{~min}$, the formation of a dominant flow channel in Ketton becomes visible; it is evident that this preferential channel is connected to the region where the dominant channel from Silurian exists. This implies that the preferential flow channel in Silurian dolomite has grown to the 


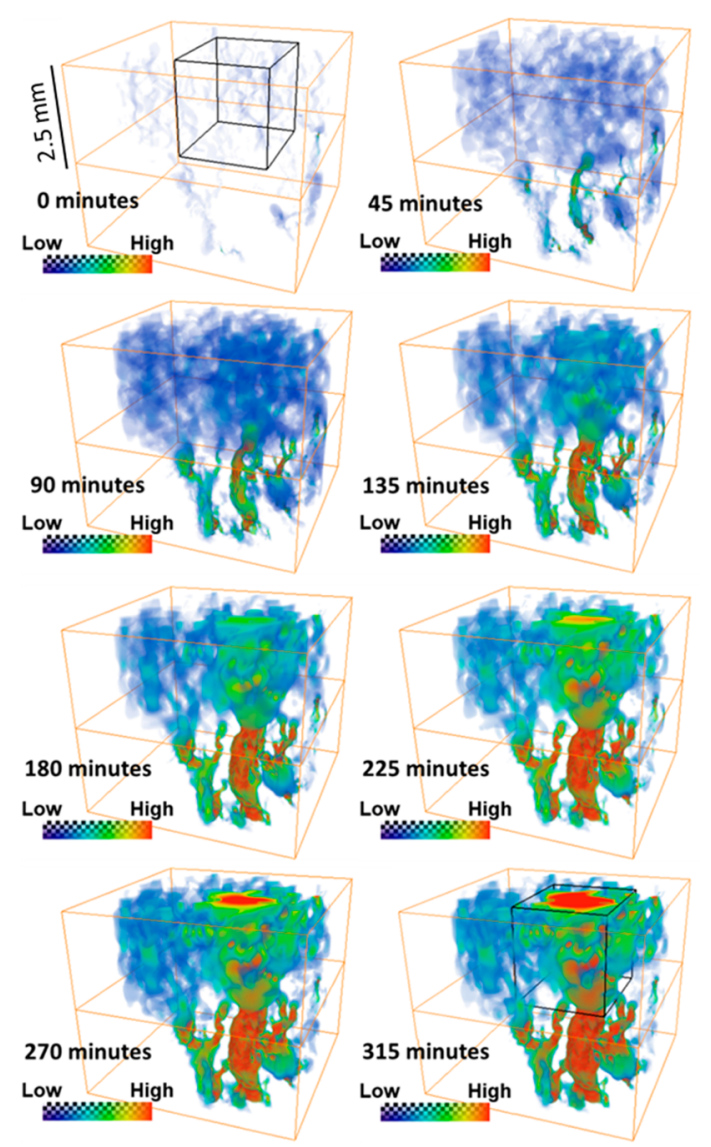

Figure 3. 3D renderings of flow velocities from modeling over the span of the experiment as dissolution advances. Silurian (dolomite) is on the bottom and Ketton (calcite) is at the top of the composite core. The direction of flow is from the bottom to top; the cooler colors represent the voxels with lower velocities, and the warmer colors represent the voxels seeing higher velocities. The full range of existing velocities (divided by the respective Darcy velocity of each image) are represented on linear color scales, customized for each image to maximize the contrast. 3D visualization indicates a two-stage dissolution in Ketton core: the first stage is characterized by a uniform growth of channels, while the second stage follows a singlechannel growth regime. This is seen as the concentrated flow in the Ketton core at later times. The black box in the first and last figures highlight the initial and final condition of the region where the dominant channel in Ketton eventually formed.

extent that it determines more focused dissolution in the calcite. This is what leads to the two-stage dissolution: the first stage is characterized by a uniform growth of channels, while the second stage follows a single-channel growth regime. The spatial memory effects in the medium with a heterogeneous flow characteristic (dolomite) are observed to change dissolution patterns in the medium with an initially morehomogeneous flow (calcite). This hitherto unobserved phenomenon implies that both different initial pore structure and dynamic changes in flow and transport characteristics in the composite core can result in a time-dependent change in dissolution patterns.

We now use velocity distributions to analyze and interpret this behavior. Figure 4 shows the plots of PDFs of velocity of the full composite, Ketton, and Silurian cores for all XMT scans.

From the PDFs of velocities in Figure 4, we observe that the average velocity of the dolomite is greater than that for the calcite for all experimental times; this is due to the lower porosity of the dolomite. As dissolution progresses, porosity increases in both rocks resulting in a decrease in their average velocity. In Figure 4B, we see a rapid change in the PDFs of velocity for Silurian dolomite at early times up to $t=45 \mathrm{~min}$ characterized by a narrowing of the distribution and a significant loss of stagnant voxels. This is also observed in Figure 3 for the dolomite part of the composite at $t=45 \mathrm{~min}$, which shows that new preferential flow channels have formed. In general, there is little change in the PDFs of velocity after $t=$ $45 \mathrm{~min}$, which is consistent with a channel widening regime throughout the experiment. The formation of a small second peak in the Silurian dolomite plot by $t=90 \mathrm{~min}$ represents the establishment of another channel, which can also be seen in Figure 3. For Ketton calcite, we observe two remarkable features: first, there is a decrease in average velocity represented by the peak from $t=0 \mathrm{~min}$ to $t=180 \mathrm{~min}$. Secondly, from $t=$ $180 \mathrm{~min}$, a second smaller peak emerges with velocities higher than the average. The first observation is related to the dissolution driven by widening of a number of channels, which results in the higher porosity throughout the sample; for the constant Darcy flow rate throughout the experiment, this leads to a decrease in average velocity. The second observation at the later times can be associated with reaction, which proceeds as the growth of a single dominant flow channel.

The nature of initial structural heterogeneity of Silurian dolomite leads to a heterogeneous initial flow characteristic (as described in section 2.3), which at a high flow rate results in the formation and growth of a few large channels, one of which dominates the flow, as evident in Figure 3. However, for the initially homogeneous pore structure and flow characteristic of Ketton calcite, Menke et al. ${ }^{29}$ showed that, under similar transport and reactive conditions (Pe ranging from 2100 to
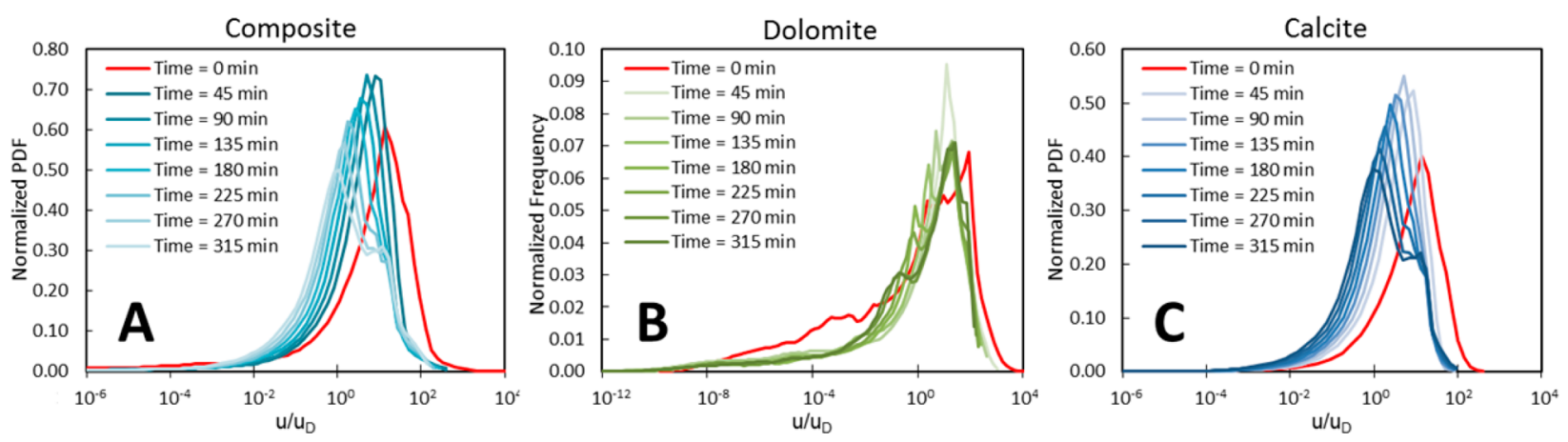

Figure 4. PDFs of the velocity over time for the composite (A), Ketton calcite (B), and Silurian dolomite (C) portions plotted separately. 

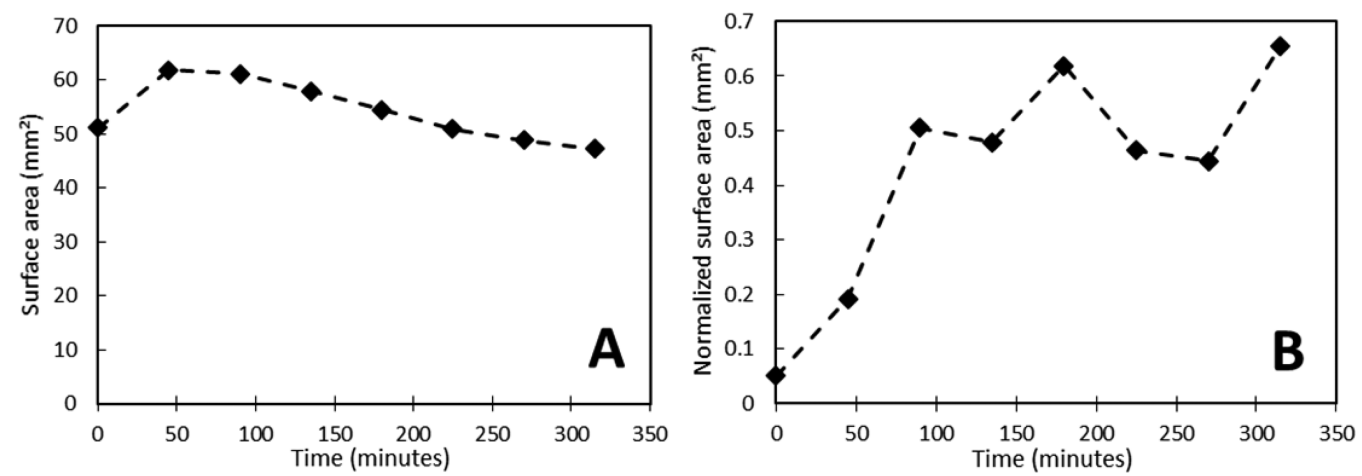

Figure 5. Plot A shows the surface area of the Ketton calcite subvolume, highlighted by a black box in Figure 3, through time; the initial increase reflects uniform dissolution of grains, and the later drop in surface area can be explained as small pores merging into one another to form a larger channel. Plot B shows the normalized surface area (surface area divided by the number of pores) against time. The 90 min point marks the establishment of a single dominant channel.

1050 and Da from $2.8 \times 10^{-5}$ to $6.5 \times 10^{-5}$ ), dissolution occurs through a widening of the existing fast-flow channels. In the experiments presented here, dissolution patterns in the Ketton pore space are additionally influenced by the dynamic change in flow heterogeneity of the preceding Silurian dolomite mineral, leading to a two-stage dissolution: initially there is a widening of the fast flow channels throughout the Ketton core, while at later times dissolution is focused through a widening of a single channel. These observations serve as a demonstration of complexity of dissolution patterns in mixed mineralogy systems. To further elucidate this phenomenon and more accurately define the time threshold when the dominant channel in the Ketton formed, we have extracted a subvolume for the Ketton rock, where the main channel has developed, as marked by the black box in Figure 3. Figure S5 shows the plot of the porosity profile of this subvolume (A) and the effective perimeter of the porosity per image slice with time (B). The perimeter was calculated for every $2 \mathrm{D}$ image slice perpendicular to the direction of flow using the Crofton formula, ${ }^{64}$ which counts the number of image line intersections to estimate curve lengths. The porosity profile increases evenly across the length of the core, suggesting that no wormholing occurs but that channel expansion does. Figure S6 shows the growth of Ketton pores at the top and side cross-sections of the subvolume analyzed up to the time when they merge to form a single channel. The images reaffirm the widening of existing flow paths, rather than etching a new path through the rock. ${ }^{14,29}$

Also within this subvolume, we calculate the area available for reaction as the sum of pore and rock voxel common interfaces. In Figure 5A, we plot the evolution of the surface area with time: the surface area increases until $t=45 \mathrm{~min}$ and then steadily decreases. With this plot alone, the point at which the dominant channel formed may not be determined because it gives little information on the effect that merging pores have on surface area change.

Recalling Figure 4C, by $t=315 \mathrm{~min}$ in the PDFs of velocity of Ketton, the second peak (representing velocities higher than average) emerges, which indicates the existence of a single channel. To mark the start of a single channel development, we resort to the analysis presented in Figure 5B. It shows the surface area normalized by the number of pores in the image. From $t=0 \mathrm{~min}$ to $t=90 \mathrm{~min}$, there is a clear increase in normalized surface area. Within this period, the increase of normalized surface area is due to the growth of individual pores. Starting from $t=90 \mathrm{~min}$, many small pores merge and a single large pore, or channel, forms. This is indicated by the fluctuation in the plot of the normalized surface area after 90 min. This method helps to identify the time at which a preferential flow path was generated at $t=90 \mathrm{~min}$.

Our combined pore-scale imaging and modeling approach allows us to study the coupled impact of structure and transport properties on the effective reaction rates in a chemically heterogeneous composite rock made up of Silurian dolomite and Ketton limestone arranged in series. The advantage of this approach is that it allows us to study complex media composed of minerals with contrasting flow, transport, and reaction characteristics. Effective reaction rates for the two minerals were shown to be in a good agreement when comparing measurements and analysis from images and from effluent analysis, thus providing a validation method for mixed mineralogy samples.

We observe a large change in the ratios of effective individual reaction rates in the dolomite and limestone (calcite). The effective reaction rates for calcite and dolomite are 1 order of magnitude lower than their corresponding batch reaction rates. The ratio of the effective reaction rates for calcite and dolomite decreases with time. We find that physical heterogeneity exerted a greater influence on the effective reaction rates than the difference in the intrinsic reaction rates for the two minerals; this was a consequence of dynamic changes in pore structure, which, through the widening of existing flow channels, affected dynamic transport rates, as clearly seen from the changing velocity distributions.

Furthermore, a detailed analysis of the dynamics of flow and transport in each mineral core has led to new observations of dissolution patterns in the calcite influenced by the preceding dolomite. Velocity field visualization and PDFs of velocity confirmed that the evolving dolomite flow heterogeneity characteristic has a large impact on the nature of dissolution in the calcite. We observe two-stage dissolution. Initially, dissolution in the more heterogeneous dolomite is concentrated in a few fast channels, whereas the Ketton dissolution is more uniform. In the second stage, a dominant channel emerges in the dolomite, providing a focused ingress of reactant to the Ketton, which too then sees dissolution concentrated in a single dominant channel. Hence, we demonstrate that the propagation of single-channel growth can occur through two minerals with contrasting flow, transport, and reaction characteristics, which in this way can preserve spatial memory effects. These experimentally observed dynamic effects, leading 
to different type of dissolution, need to be considered in poreto-field-scale modeling of reactive transport.

Our combined pore-scale imaging and modeling methodology can be extended to study reactive transport dynamics in multimineral subsurface media encountered in contaminant transport, CCS, nuclear waste disposal, and oil-recovery applications.

\section{ASSOCIATED CONTENT}

\section{S Supporting Information}

The Supporting Information is available free of charge on the ACS Publications website at DOI: 10.1021/acs.est.6b06224.

Additional experimental methodology. Figures showing a core composite assembly schematic and a $2 \mathrm{D}$ projection of the core, the experimental apparatus and assembled carbon fiber core holder, changes in a center slide of each mineral, image-derived porosity results, porosity profiles and effective perimeters of the porosity, and a series of identical 2D slices at different times. A table showing image analysis results. (PDF)

\section{AUTHOR INFORMATION}

\section{Corresponding Author}

*Phone: +44 (0)20 7594 9982; fax: +44 (0)20 7594 7444; email: q.lin11@imperial.ac.uk.

\section{ORCID}

Qingyang Lin: 0000-0001-5691-9532

\section{Author Contributions}

The manuscript was written through contributions of all authors. All authors have given approval to the final version of the manuscript.

\section{Notes}

The authors declare no competing financial interest.

\section{ACKNOWLEDGMENTS}

We gratefully acknowledge funding from the Qatar Carbonates and Carbon Storage Research Centre (QCCSRC), provided jointly by Qatar Petroleum, Shell, and Qatar Science \& Technology Park. The images acquired in this study can be downloaded from http://dx.doi.org/10.6084/m9.figshare. 4765300 .

\section{REFERENCES}

(1) Bachu, S. Sequestration of $\mathrm{CO} 2$ in geological media: Criteria and approach for site selection in response to climate change. Energy Convers. Manage. 2000, 41 (9), 953-970.

(2) Wang, Y.; Hill, a. D.; Schechter, R. S. The Optimum Injection Rate for Matrix Acidizing of Carbonate Formations. Proceedings of SPE Annual Technical Conference and Exhibition; Society of Petroleum Engineers: Richardson, TX, 1993.

(3) Zheng, C.; Bennett, G. Applied Contaminant Transport Modeling; Wiley-Interscience: New York, 1995; Vol. 2.

(4) Lin, Q.; Neethling, S. J.; Courtois, L.; Dobson, K. J.; Lee, P. D. Multi-scale quantification of leaching performance using X-ray tomography. Hydrometallurgy 2016, 164, 265-277.

(5) D’Alessandro, D. M.; Smit, B.; Long, J. R. Carbon dioxide capture: Prospects for new materials. Angew. Chem., Int. Ed. 2010, 49 (35), 6058-6082.

(6) Peng, C.; Crawshaw, J. P.; Maitland, G. C.; Trusler, J. P. M. Kinetics of calcite dissolution in $\mathrm{CO} 2$-saturated water at temperatures between $(323$ and 373$) \mathrm{K}$ and pressures up to $13.8 \mathrm{MPa}$. Chem. Geol. 2015, 403 (0), 74-85.
(7) Morse, J. W.; Mackenzie, F. T. Geochemistry of Sedimentary Carbonates, Developments in Sedimentology; Elsevier: Amsterdam, The Netherlands, 1990; Vol. 48.

(8) Swoboda-Colberg, N. G.; Drever, J. I. Mineral dissolution rates in plot-scale field and laboratory experiments. Chem. Geol. 1993, 105 (13), 51-69.

(9) Li, L.; Peters, C. A.; Celia, M. A. Upscaling geochemical reaction rates using pore-scale network modeling. Adv. Water Resour. 2006, 29 (9), 1351-1370.

(10) Black, J. R.; Carroll, S. A.; Haese, R. R. Rates of mineral dissolution under CO2 storage conditions. Chem. Geol. 2015, 399 (0), 134-144.

(11) Li, L.; Steefel, C. I.; Yang, L. Scale dependence of mineral dissolution rates within single pores and fractures. Geochim. Cosmochim. Acta 2008, 72 (2), 360-377.

(12) Gouze, P.; Luquot, L. X-ray microtomography characterization of porosity, permeability and reactive surface changes during dissolution. J. Contam. Hydrol. 2011, 120-121 (C), 45-55.

(13) Maheshwari, P.; Ratnakar, R. R.; Kalia, N.; Balakotaiah, V. 3-D simulation and analysis of reactive dissolution and wormhole formation in carbonate rocks. Chem. Eng. Sci. 2013, 90 (0), 258-274.

(14) Menke, H. P.; Andrew, M. G.; Blunt, M. J.; Bijeljic, B. Reservoir condition imaging of reactive transport in heterogeneous carbonates using fast synchrotron tomography - Effect of initial pore structure and flow conditions. Chem. Geol. 2016, 428, 15-26.

(15) Beisman, J. J.; Maxwell, R. M.; Navarre-Sitchler, A. K.; Steefel, C. I.; Molins, S. ParCrunchFlow: an efficient, parallel reactive transport simulation tool for physically and chemically heterogeneous saturated subsurface environments. Comput. Geosci. 2015, 19 (2), 403-422.

(16) Marty, N. C. M.; Bildstein, O.; Blanc, P.; Claret, F.; Cochepin, B.; Gaucher, E. C.; Jacques, D.; Lartigue, J. E.; Liu, S.; Mayer, K. U.; et al. Benchmarks for multicomponent reactive transport across a cement/clay interface. Comput. Geosci. 2015, 19 (3), 635-653.

(17) Molins, S.; Trebotich, D.; Steefel, C. I.; Shen, C. An investigation of the effect of pore scale flow on average geochemical reaction rates using direct numerical simulation. Water Resour. Res. 2012, 48 (3).10.1029/2011WR011404

(18) Steefel, C. I.; Beckingham, L. E.; Landrot, G. Micro-Continuum Approaches for Modeling Pore-Scale Geochemical Processes. Rev. Mineral. Geochem. 2015, 80 (1), 217-246.

(19) Steefel, C. I.; Appelo, C. A. J.; Arora, B.; Jacques, D.; Kalbacher, T.; Kolditz, O.; Lagneau, V.; Lichtner, P. C.; Mayer, K. U.; Meeussen, J. C. L.; et al. Reactive transport codes for subsurface environmental simulation. Comput. Geosci. 2015, 19 (3), 445-478.

(20) Steefel, C. I.; Lasaga, A. C. A coupled model for transport of multiple chemical species and kinetic precipitation/dissolution reactions with application to reactive flow in single phase hydrothermal systems. Am. J. Sci. 1994, 294 (5), 529-592.

(21) Steefel, C. I.; Maher, K. Fluid-rock interaction: A reactive transport approach. Rev. Mineral. Geochem. 2009, 70, 485-532.

(22) Daccord, G.; Lenormand, R.; Liétard, O. Chemical dissolution of a porous medium by a reactive fluid-I. Model for the "wormholing" phenomenon. Chem. Eng. Sci. 1993, 48 (1), 169-178.

(23) Fredd, C. N.; Fogler, H. S. Optimum conditions for wormhole formation in carbonate porous media: Influence of transport and reaction. SPE J. 1999, 4 (3), 196-205.

(24) Golfier, F.; Zarcone, C.; Bazin, B.; Lenormand, R.; Lasseux, D.; Quintard, M. On the ability of a Darcy-scale model to capture wormhole formation during the dissolution of a porous medium. J. Fluid Mech. 2002, 457, 213-254.

(25) Noiriel, C.; Gouze, P.; Bernard, D. Investigation of porosity and permeability effects from microstructure changes during limestone dissolution. Geophys. Res. Lett. 2004, 31 (24), 1-4.

(26) Noiriel, C.; Bernard, D.; Gouze, P.; Thibault, X. Hydraulic properties and microgeometry evolution accompanying limestone dissolution by acidic water. Oil Gas Sci. Technol. 2005, 60 (1), 177192. 
(27) Luquot, L.; Gouze, P. Experimental determination of porosity and permeability changes induced by injection of $\mathrm{CO} 2$ into carbonate rocks. Chem. Geol. 2009, 265 (1-2), 148-159.

(28) Gharbi, O.; Bijeljic, B.; Boek, E. S.; Blunt, M. J. Changes in Pore Structure and Connectivity Induced by $\mathrm{CO} 2$ Injection in Carbonates: A Combined Pore-Scale Approach. Energy Procedia 2013, 37 (0), 5367-5378.

(29) Menke, H. P.; Bijeljic, B.; Andrew, M. G.; Blunt, M. J. Dynamic three-dimensional pore-scale imaging of reaction in a carbonate at reservoir conditions. Environ. Sci. Technol. 2015, 49 (7), 4407-4414.

(30) Mercado, A. The kinetics of mineral dissolution in carbonate aquifers as a tool for hydrological investigations, II. Hydrogeochemical models. J. Hydrol. 1977, 35 (3-4), 365-384.

(31) Hao, Y.; Smith, M.; Sholokhova, Y.; Carroll, S. CO2-induced dissolution of low permeability carbonates. Part II: Numerical modeling of experiments. Adv. Water Resour. 2013, 62, 388-408.

(32) Smith, M. M.; Sholokhova, Y.; Hao, Y.; Carroll, S. A. CO2induced dissolution of low permeability carbonates. Part I: Characterization and experiments. Adv. Water Resour. 2013, 62, 370-387.

(33) Ellis, B.; Peters, C.; Fitts, J.; Bromhal, G.; McIntyre, D.; Warzinski, R; Rosenbaum, E. Deterioration of a fractured carbonate caprock exposed to CO 2-acidified brine flow. Greenhouse Gases: Sci. Technol. 2011, 1 (3), 248-260.

(34) Deng, H.; Molins, S.; Steefel, C.; DePaolo, D.; Voltolini, M.; Yang, L.; Ajo-Franklin, J. A 2.5D Reactive Transport Model for Fracture Alteration Simulation. Environ. Sci. Technol. 2016, 50 (14), $7564-7571$.

(35) Carroll, S. A.; McNab, W. W.; Dai, Z.; Torres, S. C. Reactivity of Mount Simon Sandstone and the Eau Claire Shale Under $\mathrm{CO} 2$ Storage Conditions. Environ. Sci. Technol. 2013, 47 (1), 252-261.

(36) Gao, J.; Xing, H.; Tian, Z.; Pearce, J. K.; Sedek, M.; Golding, S. D.; Rudolph, V. Reactive transport in porous media for $\{\mathrm{CO} 2\}$ sequestration: Pore scale modeling using the lattice Boltzmann method. Comput. Geosci. 2017, 98, 9-20.

(37) Salehikhoo, F.; Li, L. The role of magnesite spatial distribution patterns in determining dissolution rates: When do they matter? Geochim. Cosmochim. Acta 2015, 155, 107-121.

(38) Li, L.; Salehikhoo, F.; Brantley, S. L.; Heidari, P. Spatial zonation limits magnesite dissolution in porous media. Geochim. Cosmochim. Acta 2014, 126, 555-573.

(39) Vandeginste, V.; John, C. M. Influence of climate and dolomite composition on dedolomitization: insights from a multi-proxy study in the central Oman Mountains. J. Sediment. Res. 2012, 82, 177-195.

(40) Scholle, P. A.; Ulmer-Scholle, D. S.; Geologists, A. A. P. A Color Guide to the Petrography of Carbonate Rocks: Grains, Textures, Porosity, Diagenesis, AAPG Memoir 77; American Association of Petroleum Geologists: Tulsa, OK, 2003.

(41) Blunt, M. J.; Bijeljic, B.; Dong, H.; Gharbi, O.; Iglauer, S.; Mostaghimi, P.; Paluszny, A.; Pentland, C. Pore-scale imaging and modelling. Adv. Water Resour. 2013, 51 (0), 197-216.

(42) Cnudde, V.; Boone, M. N. High-resolution X-ray computed tomography in geosciences: A review of the current technology and applications. Earth-Sci. Rev. 2013, 123, 1-17.

(43) Dhawan, N.; Safarzadeh, M. S.; Miller, J. D.; Moats, M. S.; Rajamani, R. K.; Lin, C. L. Recent advances in the application of X-ray computed tomography in the analysis of heap leaching systems. Miner. Eng. 2012, 35 (0), 75-86.

(44) Ketcham, R. a; Carlson, W. D. Acquisition, optimiziation and interpretation of $\{\mathrm{X}\}$-ray computed tomography imagery: applications to the geosciences. Comput. Geosci. 2001, 27 (4), 381-400.

(45) Lai, P.; Krevor, S. Pore scale heterogeneity in the mineral distribution and surface area of Berea sandstone. Energy Procedia 2014, 63 (0), 3582-3588.

(46) Lin, Q.; Al-Khulaifi, Y.; Blunt, M. J.; Bijeljic, B. Quantification of sub-resolution porosity in carbonate rocks by applying high-salinity contrast brine using X-ray microtomography differential imaging. $A d v$. Water Resour. 2016, 96, 306-322.

(47) Ott, H.; Andrew, M.; Blunt, M.; Snippe, J. Capillary-Driven Solute Transport and Precipitation in Porous Media during Dry-Out.
In Society of Core Analysis Conference Paper SCA2014-25; 2014; Vol. 16, p 10046.

(48) Wildenschild, D.; Sheppard, A. P. X-ray imaging and analysis techniques for quantifying pore-scale structure and processes in subsurface porous medium systems. Adv. Water Resour. 2013, 51, 217-246.

(49) Andrew, M.; Bijeljic, B.; Blunt, M. Reservoir Condition Porescale Imaging of Multiple Fluid Phases Using X-ray Microtomography. J. Visualized Exp. 2015, 96 (96), 1-10.

(50) Luhmann, A. J.; Kong, X.; Tutolo, B. M.; Garapati, N.; Bagley, B. C.; Saar, M. O.; Seyfried, W. E., Jr Experimental dissolution of dolomite by $\mathrm{CO} 2$-charged brine at $100^{\circ} \mathrm{C}$ and 150 bar: Evolution of porosity, permeability, and reactive surface area. Chem. Geol. 2014, 380 (0), 145-160.

(51) Ahr, W. M. Geology of Carbonate Reservoirs: The Identifi Cation, Description, And Characterization of Hydrocarbon Reservoirs in Carbonate Rocks; Wiley: Hoboken, NJ, 2008.

(52) Andrew, M.; Bijeljic, B.; Blunt, M. J. Pore-scale imaging of geological carbon dioxide storage under in situ conditions. Geophys. Res. Lett. 2013, 40 (15), 3915-3918.

(53) Alotaibi, M.; Azmy, R.; Nasr-El-Din, H. Wettability Challenges in Carbonate Reservoirs. In SPE Improved Oil Recovery Symposium; Society of Petroleum Engineers: Richardson, TX, 2010; pp 24-28.

(54) Peng, C. Chemical Interactions between Carbon Dioxide Acidified Aqueous Fluids and Carbonate Minerals. Ph.D Dissertation, Imperial College London: London, 2015.

(55) Raeini, A. Q.; Blunt, M. J.; Bijeljic, B. Modelling two-phase flow in porous media at the pore scale using the volume-of-fluid method. J. Comput. Phys. 2012, 231 (17), 5653-5668.

(56) Bijeljic, B.; Raeini, A.; Mostaghimi, P.; Blunt, M. J. Predictions of non-Fickian solute transport in different classes of porous media using direct simulation on pore-scale images. Phys. Rev. E - Stat. Nonlinear, Soft Matter Phys. 2013, 87 (1), 13011.

(57) Bijeljic, B.; Mostaghimi, P.; Blunt, M. J. Insights into nonFickian solute transport in carbonates. Water Resour. Res. 2013, 49 (5), 2714-2728.

(58) Buades, A.; Coll, B.; Morel, J. M. A Non-Local Algorithm for Image Denoising. In 2005 IEEE Computer Society Conference on Computer Vision and Pattern Recognition CVPR05; 2005; Vol. 2, pp 60-65.10.1109/CVPR.2005.38

(59) Schlüter, S.; Sheppard, A.; Brown, K.; Wildenschild, D. Image processing of multiphase images obtained via X-ray microtomography: A review. Water Resour. Res. 2014, 50 (4), 3615-3639.

(60) Lasaga, A. C. Chemical Kinetics of Water-Rock Interactions. J. Geophys. Res. solid earth 1984, 89 (B6), 4009-4025.

(61) Péclet, J. C. E. Traité de l'éclairage; La Librairie scientifique et Industielle de Malher et Cie: Paris, France, 1827.

(62) Mostaghimi, P.; Bijeljic, B.; Blunt, M. Simulation of flow and dispersion on pore-space images. SPE J. 2012, 17, 1131-1141.

(63) Andrew, M.; Bijeljic, B.; Blunt, M. J. Pore-scale imaging of trapped supercritical carbon dioxide in sandstones and carbonates. Int. J. Greenhouse Gas Control 2014, 22 (0), 1-14.

(64) Crofton, M. W. On the Theory of Local Probability, Applied to Straight Lines Drawn at Random in a Plane; The Methods Used Being Also Extended to the Proof of Certain New Theorems in the Integral Calculus. Philos. Trans. R. Soc. London 1868, 158 (May), 181-199. 